\title{
AN ASYMPTOTIC COMPARISON OF SUBSET SELECTION PROCEDURES ${ }^{*}$
}

by

Milton Sobel and S. P. Yen

Technical Report 118

University of Minnesota

Minneapolis, Minnesota

* This research was supported by National Science Foundation Grant GP-9018. 
An Asymptotic Comparison of Subset Selection Procedures

by Milton Sobel and S. P. Yen

University of Minnesota

A procedure $R$ for selecting a subset of $k$ populations containing at least one of the $t$ best populations was introduced in [2]. For normal populations we put $\pi_{i}$ in the selected subset if and only if $\bar{x}_{i} \geq \bar{x}_{[k-s+1]}-a_{s}$, where $\bar{x}_{i}$ is the sample mean from $\Pi_{i}$ and the ordered sample means are $\bar{x}_{[1]} \leq \cdots \leq \bar{x}_{[k]}$. Under procedure $R$ both $s \geq 1$ and $a_{s} \geq 0$ are determined so that the probability of a correct subset $P\{C S\} \geq P^{*} \quad$ (specified), whenever the minimum difference between any one of the $t$ largest population means and any one of the $k-t$ smallest is at least $\delta^{*}$ (specified). For $t=1$ and $\delta^{*}=0$ the goal is the same as that considered by Gupta [1], but his procedure $R_{G}$ is not the same as procedure $R$. In [2] many exact and asymptotic comparisons are made for $t \geq 1$ and $\delta^{*} \geq 0$ but the emphasis there is on the equal parameter (EP) configuration, where the expected subset size is maximized. Moreover for $t=1$ and $\delta^{*}=0$ the value of the expected subset sizes $E\{S \mid E P\}$ is the same, namely $\mathrm{kP}^{*}$, for both procedures and hence this criteria does not lead to any clear preference in this special case. It was shown in [2] that if either $t>1$ or $\delta^{*}>0$ then asymptotically $\left(\mathrm{P}^{*} \rightarrow 1\right)$ the value of $E\{S \mid E P\}$ is smaller for procedure $R$ than for the natural generalization $R_{M}$ of procedure $R_{G}$ for $t>1$ and $\delta^{*}>0$; in fact, the ratio approaches zero as $P^{*} \rightarrow 1$.

In this note we consider only the special case $t=1$ and $\delta^{*}=0$ and make asymptotic $\left(P^{*} \rightarrow 1\right)$ comparisons of $E\{S \mid \underline{\theta}\}$ for any k-vector $\theta$ of true parameter values. Let $\theta_{[1]} \leq \ldots \leq \theta_{[k]}$ denote the ordered 
parameter values and let $\delta_{i j}=\theta_{[i]}-\theta_{[j]}$. In terms of the differences $\delta_{i j}$, we find the exact sets $S_{R}$ and $s_{G}$ of vectors $\underline{\theta}$ which have a smaller asymptotic $\left(P^{*} \rightarrow 1\right)$ value for $E\{S \mid \theta\}$ under procedure $R$ and $R_{G}$, respectively. Since both $S_{R}$ and $S_{G}$ are non-empty, it follows that neither of these two procedures is uniformly better than the other in the sense of this criterion. We assume normal populations with a common variance $\sigma^{2}$, which we can take to be unity.

Under procedure $R$ with $t=1$, we set $s=k-1$ for $P^{*}$ close to one and obtain for $\delta^{*}=0$

$$
\begin{aligned}
& E\{s \mid \underline{\theta}, R\}=\sum_{i=1}^{k} P\left\{\bar{x}_{(i)} \geq \bar{x}_{[2]}-a_{k-1}\right\} \\
& =\sum_{i=1}^{k}\left[1-\sum_{\substack{j=1 \\
\neq i}}^{k} P\left\{\bar{x}_{(i)}+a_{k-1}<\bar{x}_{(j)}, \bar{x}_{(j)}=\bar{x}_{[2]}\right\}\right]
\end{aligned}
$$

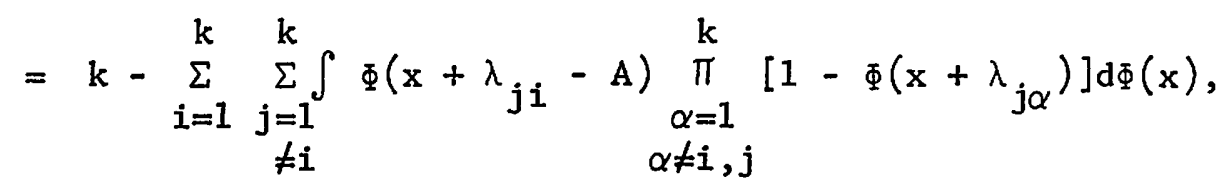

where $A=a_{k-1} \sqrt{n}, \lambda_{i j}=\delta_{i j} \sqrt{n}$ and $\Phi(x), \varphi(x)$ are used to denote the standard normal c.d.f。 and density, respectively. As in Section 8 of [2] we use the Laplace-Feller expansion for the 'tail' of the normal c.d.f. in (1), drop the denominator and then 'complete the square'. Neglecting the error term, $o\left(\exp \left\{-\theta^{2}\left(A-\lambda_{k 1}\right)^{2} / 2\right\}\right)$, we obtain

$$
\begin{aligned}
k-E\{s \mid \underline{\theta}, R\} & \approx \frac{C}{A} \sum_{i} \sum_{j \neq i} \int\left[\varphi\left(x+A-\lambda_{j i}\right) \varphi(x)\right] \prod_{\alpha=1}^{k} \Phi\left(x-\lambda_{j \alpha}\right) d x \\
& \approx \frac{C}{A} \sum_{i} \sum_{j \neq i} \varphi\left(\frac{A-\lambda_{j i}}{\sqrt{2}}\right) \int \prod_{\substack{\alpha=1 \\
\alpha \neq i, j}}^{k} \Phi\left(\frac{y}{\sqrt{2}}+D_{\alpha}\right) d \Phi(y)
\end{aligned}
$$




$$
\begin{aligned}
& =\frac{\mathrm{C}}{\mathrm{A}^{\mathrm{k}-1}} \sum_{i} \sum_{j \neq i} \varphi\left(\frac{\mathrm{A}-\lambda_{j i}}{\sqrt{2}}\right) \int\left[\prod_{\substack{\alpha=1 \\
\alpha \neq i, j}}^{k} \varphi\left(\frac{\mathrm{y}}{\sqrt{2}}+\mathrm{D}_{\alpha}\right)\right] \varphi(y) d y \\
& \approx \frac{c}{A^{k-1}} \sum_{i} \sum_{j \neq i} \varphi\left(\frac{A-\lambda j i}{\sqrt{2}}\right) \quad \exp \left\{-\left[\sum_{\alpha \neq i, j} D_{\alpha}^{2}-\frac{1}{k}\left(\sum_{\alpha \neq i, j} D_{\alpha}\right)^{2}\right] / 2\right\}
\end{aligned}
$$

where $D_{\alpha}=\left(\lambda_{j i}-A-2 \lambda_{j \alpha}\right) / 2$. Collecting the factors of the form $\exp \left\{-C A^{2}\right\}$ and $\exp \{C A\}$, we use the fact that

$$
(k-1) \lambda_{j i}-\sum_{\alpha \neq i, j} \lambda_{j \alpha}=\lambda_{j i}+\sum_{\alpha \neq j} \lambda_{\alpha i}=\sum_{\alpha} \lambda_{\alpha i}
$$

does not depend on $j$, and obtain from (2)

$$
\begin{aligned}
k-E\{s \mid \underline{\theta}, R\} & \approx \frac{C}{A^{k-1}} \sum_{i} \sum_{j \neq i} e^{-\left(\frac{k-1}{2 k}\right)\left(A-\lambda_{j i}\right)^{2}} \exp \left\{\frac{l}{k}\left(\lambda_{j i}-A\right) \sum_{\alpha \neq i, j} \lambda_{j \alpha}\right\} \\
& \approx \frac{C}{A^{k-1}} e^{-\left(\frac{k-1}{2 k}\right) A^{2}} \sum_{i} \exp \left\{\frac{A}{k} \sum_{\alpha} \lambda_{\alpha i}\right\} .
\end{aligned}
$$

The maximum term in (4) for large $A$ is obtained by maximizing over $i$ the sum in braces and this clearly occurs for $i=1$. Hence

$$
\mathrm{k}-\mathrm{E}\{\mathrm{s} \mid \underline{\theta}, \mathrm{R}\} \approx \frac{\mathrm{C}}{\mathrm{A}^{\mathrm{k}-1}} \exp \left\{-\left(\frac{\mathrm{k}-1}{2 \mathrm{k}}\right) \mathrm{A}^{2}+\frac{\mathrm{A}}{\mathrm{k}} \sum_{\alpha} \lambda_{\alpha 1}\right\}
$$

It is shown in (8.11) of [2] that for $P^{*} \rightarrow 1$

$$
A=\sqrt{2\left(\frac{\mathrm{k}}{\mathrm{k}-1}\right) \ln \left(\frac{1}{1-\mathrm{p}^{*}}\right)}
$$

and applying this to (5) gives the final form for procedure $R$

$$
\mathrm{k}-\mathrm{E}\{\mathrm{S} \mid \underline{\theta}, \mathrm{R}\} \approx \frac{\mathrm{C}}{\mathrm{A}^{\mathrm{k}-1}}\left(1-\mathrm{P}^{*}\right) \exp \left\{\left(\sum_{\alpha} \lambda_{\alpha 1}\right) \sqrt{\frac{2}{\mathrm{k}(\mathrm{k}-1)} \ln \left(\frac{1}{1-\mathrm{P}^{*}}\right)}\right\}
$$


For procedure $R_{G}$ it is easily shown as in Gupta [1] that

$$
\begin{aligned}
E\left\{S \mid \underline{\theta}, R_{G}\right\} & =\sum_{i=1}^{k} \int_{j \neq i} \prod_{j} \Phi\left(x+A+\lambda_{i j}\right) \mathrm{d} \Phi(x) \\
& \approx \sum_{i} \int\left\{1-\sum_{j \neq i}\left[1-\Phi\left(x+A+\lambda_{i j}\right)\right]\right\} \mathrm{d} \Phi(x) \\
& =k-\sum_{i j \neq i}\left[1-\Phi\left(\frac{A+\lambda_{i j}}{\sqrt{2}}\right)\right]
\end{aligned}
$$

Hence

$$
\begin{aligned}
k-E\left\{s \mid \underline{\theta}, R_{G}\right\} & \approx \frac{C}{A} \sum_{i} \sum_{j \neq i} \varphi\left(\frac{A+\lambda_{i j}}{\sqrt{2}}\right) \\
& \approx \frac{C}{A} e^{-A^{2 / 4}} \sum_{i j \neq i} \sum_{j \neq i} e^{A \lambda} j / 2
\end{aligned}
$$

The maximum term for large $A$ is obtained by setting $j=k$ and $i=1$; hence this gives

$$
\mathrm{k}-\mathrm{E}\left\{\mathrm{s} \mid \underset{\theta}{\underline{\theta}}, \mathrm{R}_{\mathrm{G}}\right\} \approx \frac{\mathrm{C}}{\mathrm{A}} \exp \left\{-\frac{\mathrm{A}^{2}}{4}+\frac{\mathrm{A}}{2} \lambda_{\mathrm{k} 1}\right\}
$$

In (8.11) of [2] we set $s=t=1$ to obtain the A-value for procedure $R_{G}$, namely

$$
A=2 \sqrt{\ln \left(\frac{1}{1-P^{*}}\right)}
$$

and applying this to (10) gives the final form

$$
\mathrm{k}-\mathrm{E}\left\{\mathrm{s} \mid \underline{\theta}, \mathrm{R}_{\mathrm{G}}\right\} \approx \frac{\mathrm{C}}{\mathrm{A}}\left(1-\mathrm{P}^{*}\right) \exp \left\{\lambda_{\mathrm{k} 1} / \sqrt{\ln \left(\frac{1}{1-\mathrm{P}^{* *}}\right)}\right\} .
$$

It follows from (7) and (12) that $E\{S \mid \underline{\theta}, \mathrm{R}\}$ is smaller than $E\left\{S \mid \underline{\theta}, R_{G}\right\}$ for $P^{*}$ close to one when

$$
\sqrt{\frac{2}{k(k-1)}} \sum_{\alpha} \lambda_{\alpha 1}>\lambda_{k 1}
$$

and it is larger when the inequality is reversed. For $k=2$ the procedures are identical and (13) is vacuous. For $k=3$ the inequality 
in (13) holds when

$$
\delta_{21}>(1+\sqrt{3}) \delta_{32}=(2.732 \ldots) \delta_{32}
$$

If we define the configuration $c_{j}(j=1,2, \ldots, k)$ by setting

$$
\theta_{[1]}=\cdots=\theta_{[k-j]} ; \theta_{[k-j+1]}=\ldots=\theta_{[k]}
$$

then (13) takes the form

$$
j>\sqrt{\frac{k(k-1)}{2}}
$$

and we note that (13) always holds for $c_{k-1}$ for $k>2$. On the other hand, for all k>2 the inequality in (13) is reversed for $C_{1}$ and also for the configuration in which adjacents parameters are equally spaced. A table of values for $E\left\{s \mid C_{j}\right\}(j=1,2,3,4)$ for $k=5$ is included in [3] and it illustrates numerically the results proved above.

\section{$\underline{\text { References }}$}

[1] Gupta, S. S. (1965). On some multiple decision (selection and ranking) rules. Technometrics $\underset{7}{=} 225-245$.

[2] Sobel, M. (1969). Selecting a subset containing at least one of the t best populations. Multivariate Analysis Vol. II Proceedings of an International Symposium. Ed. by P. R. Krishnaiah. Academic Press, New York.

[3] Yen, S. P. (1969). Efficiency Comparisons for Two Subset Selection Procedures. University of Minnesota, Technical Report No. 119. 